\title{
ESPAÑA DESDE EUROPA: PERCEPCIONES Y RELACIONES (1982-2004)
}

\author{
Spain Seen from Europe. Perceptions and \\ Relations (1982-2004)
}

\author{
Marco Mugnaini \\ Universidad de Pavía - Italia \\ E-mail: marco.mugnaini@unipv.it
}

0 Autor

FIRMA INVITADA

En este trabajo se estudia la relación entre España y las políticas europeas en el periodo 19822004, utilizando como enfoque la historia de las relaciones internacionales. En el periodo examinado aquí, el sistema internacional se caracterizó por grandes cambios, pasando de la "segunda guerra fría” de los años ochenta a las nuevas dinámicas de la posguerra fría en los años noventa y luego en 2001-2003; cambios que han involucrado a Europa en general (transición de la CEE a la UE, políticas de ampliación, proyecto Euro-Med, UEM, etc.) y a España en particular. La trayectoria histórica de la relación entre España y Europa es analizada en esa compleja fase de transformación del sistema internacional. Las percepciones que los europeos han tenido de España también se examinan con respecto a los diferentes Gobiernos que se sucedieron en Madrid desde el periodo de transición hasta 2004. Se dedica atención a los principales protagonistas de los eventos analizados, en particular por su papel en la política internacional.

España; relaciones internacionales; integración europea; OTAN; Asociación Euro-Mediterránea.

Q Key mords

Central African Republic; conflict; peace; Spain; international relations; european integration; NATO; Euro-Mediterranean Partnership. 
This paper studies the relationship between Spain and European policies in the period 1982-2004, using a history of international relations approach. In the period examined here, the international system was characterized by great changes, passing from the "second cold war" of the eighties to the new dynamics of the post-cold war of the nineties and then of 2001-2003; changes that involved Europe in general (transition from the EEC to the EU, enlargement policies, Euro-Med project, etc.) and Spain in particular. The historical trajectory of the relationship between Spain and Europe is analyzed in this complex phase of transformation of the international system. The perceptions that the Europeans have had of Spain are also examined with respect to the different governments that succeeded each other in Madrid from the transition period until 2004. Specific attention is dedicated to the main protagonists of the events analyzed, particular for their role in international politics.

\section{Introducción}

El tema de mi intervención es un desafío por varias razones, a partir de la amplitud cronológica y la complejidad de los temas involucrados, por lo tanto, lo enfocaré de manera pragmática. Para empezar, debemos poner algunos hitos con respecto a la periodización adoptada, en especial el término a quo, el año 1982, y el término ad quem, el año 2004: dos años que pueden presentar elementos semejantes como fechas de periodización, pero también aspectos diferentes dependiendo de si los observamos desde el punto de vista de España o del resto de Europa. Al respecto, aclaro desde ya que, analizando el periodo comprendido entre 1982 y 2004, hablaré de España y de los acontecimientos políticos españoles, y de las iniciativas diplomáticas en las que España fue protagonista o participante destacada. Pero lo haré tratando de reconstruir también el punto de vista de los demás países europeos, tratando de comprender cómo los europeos percibieron y se relacionaron con la política española de aquellos años; además lo haré adoptando metodológicamente una perspectiva de historia de las relaciones internacionales ${ }^{1}$.

Sabemos que España y el resto de Europa están incluidas en un sistema internacional en constante transformación. Ese mismo sistema internacional que, entre 1982 y 2004, experimentó numerosos cambios, de los cuales el más destacado es, sin duda, la transición entre el sistema bipolar de la guerra fría, que terminó entre 1990 (año de la reunificación alemana) y 1991 (año de la disolución de la Unión Soviética), y el sistema internacional de la posguerra fría. Es notorio que en 1982 (nuestro término a quo) España y el resto de Europa formaban parte del sistema bipolar de la que se conoce como "segunda guerra fría" ${ }^{2}$. En cambio, en 2004 (nuestro término ad quem) no solo hacía ya unos años que la guerra fría había terminado, sino que habíamos entrado en el nuevo milenio y el sistema internacional había experimentado nuevos y radicales cambios geopolíticos ${ }^{3}$. El periodo 1982-2004 se compone, pues, de varios subperiodos, cada uno de ellos caracterizado de manera autónoma o por elementos diferentes.

1 Pereira, J. C. (Coord.) (2003). Historia de las relaciones internacionales contemporáneas. 2. ${ }^{a}$ edición. Barcelona: Ariel; Best, A., Hanhimäki, J. M., Maiolo, J. A., \& Schulze, K. E. (2008). International History of the Twentieth Century and Beyond. $2^{\text {th }}$ edition. London - New York: Routledge.

2 Sobre los diferentes periodos y temas del sistema internacional de la guerra fría: Leffler, M. P. \& Westad, O. A. (Eds.) (2010). The Cambridge History of the Cold War. 3 vols. Cambridge: Cambridge University Press.

3 Lundestad, G. (Ed.) (2013). International Relations Since the End of the Cold War. Oxford: Oxford University Press; Bariè, O. (2013). Dalla guerra fredda alla grande crisi. Il nuovo mondo delle relazioni internazionali. Bologna: il Mulino. 
Estos son los datos cronológicos y el marco internacional de referencia de mi estudio y dentro de los mismos deben colocarse y analizarse tanto la política de España y sus relaciones con Europa $^{4}$, como las percepciones a las que dieron origen. También debe tenerse en cuenta que, en la historiografía española, el periodo 1982-2004 se subdivide normalmente en la fase de los gobiernos del PSOE de Felipe González (de 1982 a 1996) y en la fase de los gobiernos del PP de José María Aznar (de 1996 a 2004)후 una subdivisión que, como veremos, corresponde solo en parte a la periodización de la historiografía internacional que es privilegiada aquí.

Vista la amplitud cronológica de mi ponencia, me concentraré en algunos momentos clave y en los temás de mayor importancia y, claro está, de una forma general.

\section{Los años ochenta: transición interna y nuevas coordenadas de política exterior}

Durante el periodo de la transición, la Europa comunitaria desplegó una intensa actividad tendente a favorecer el proceso de democratización y estabilización institucional en España y Portugal. En este sentido, es de destacar la particular acción del Parlamento europeo y de la Asamblea del Consejo de Europa; y en el mes de noviembre de 1977, España se convertía en el vigésimo miembro del Consejo de Europa. En el mismo año 1977, Madrid presentó la solicitud de adhesión a las comunidades europeas, pero el proceso de adhesión a la CEE resultó más complejo y la ruta que recorría España se cruzó con las rutas simultáneas de Grecia y Portugal.

Lo que se concretó luego, entre 1979 y 1986, se conoce como la segunda ampliación de la CEE, esta vez hacia la Europa meridional, después de la primera ampliación de 1973 a Gran Bretaña, Irlanda y Dinamarca ${ }^{6}$. Las adhesiones de Atenas, Lisboa y Madrid presentaban varias coincidencias y semejanzas, pero también no pocas particularidades nacionales que resultan evidentes si comparamos la ampliación helénica de 1979-1981 con la ibérica de 1985-19867. Uno de los elementos que las diferenciaban, recordamos, era la diversa posición de los tres países con respecto a la Alianza Atlántica, de la cual Portugal era miembro fundador desde 1949, mientras que Grecia había ingresado en la misma en 1952 (junto con Turquía) ${ }^{8}$. España, por

4 Para la visión histórica de la política exterior española: García Cantalapiedra, D., \& Pacheco Pardo, R. (Eds.) (2014). Contemporary Spanish Foreign Policy. London and New York: Routledge; Beneyto, J. M., y Pereira, J. C. (Dir.) (2015). Historia de la política exterior española en los siglos XX y XXI. Volumen I y II. Madrid: CEU Ediciones; Pereira, J. C. (Coord.) (2017). La política exterior de España. De 1800 hasta hoy. 2. edición. Barcelona: Ariel.

5 Prego, V. (2000). Presidentes. Veinticinco años de historia narrada por los cuatro jefes de Gobierno de la democracia. Barcelona: Plaza \& Janes; Powell, C. (2001). España en democracia, 1975-2000. Barcelona: Plaza \& Janes; Tusell, J. (Coord.) (2003). La transición a la democracia y el reinado de Juan Carlos I. Historia de España Menéndez Pidal. Dirigida por José María Jover Zamora. Tomo XLII. Madrid: Espasa Calpe; Núñez Seixas, X. M. (Coord.) (2017). España en democracia, 1975-2011. Volumen 10 Historia de España - Josep Fontana y Ramón Villares - Directores. Barcelona: Crítica - Marcial Pons.

6 Morelli, U. (2011). Storia dell'integrazione europea. Milano: Guerini; Moreno Juste, A., Núñez Peñas, V. (2017). Historia de la construcción Europea desde 1945. Madrid: Alianza.

7 Para el caso español: Pereira Castañares, J. C., \& Moreno Juste, A. (1991). La Spagna franchista di fronte al processo di costruzione europea (1945-1970). En Storia delle relazioni internazionali, a. 7, n. 1, 54-88; Bassols, $R$. (1995). España en Europa. Historia de la adhesión a la CE 1957-85. Madrid: Política Exterior; MacLellan, J. C. (2000). Spain and the Process of European Integration, 1957-85. New York: Palgrave; Moreno Juste, A. (2000). Las relaciones España/Europa en el siglo XX: notas para una interpretación. Cuadernos de Historia Contemporánea, 22 , 95-133.

8 Smith, M. (2000). NATO Enlargement during the Cold War. Basingstoke and New York: Palgrave Macmillan (pp. 62-90).
Durante el periodo de la transición, la Europa comunitaria desplegó una intensa actividad tendente a favorecer el proceso de democratización 
su parte, todavía no era miembro de la Alianza Atlántica. Además del Pacto Ibérico con Lisboa, renovado varias veces, desde 1953 Madrid basaba su seguridad sobre todo en la relación bilateral con los Estados Unidos, y mantenía una relación indirecta con la OTAN precisamente a través de ese país; mas, al afianzarse las nuevas instituciones democráticas en España, había surgido el problema de su adhesión directa a la alianza occidental9 .

En el periodo 1976-1978, para España había sido prioritario solucionar la cuestión institucional, hasta el punto de dejar relegados a un segundo plano los temas de política exterior. Pero, una vez organizadas las cuestiones internas más urgentes y con la vigencia de la nueva Constitución (diciembre de 1978), y después de las elecciones de marzo de 1979 en las que se impuso la UDC, el Gobierno Suárez-Oreja se dedicó a orientar su política exterior posterior a la Constituyente. En este contexto, la cuestión de las dos adhesiones, a la CEE y a la OTAN, se había convertido en el principal problema para el Gobierno de Madrid ${ }^{10}$. De hecho, a comienzos de la década de los ochenta, España tenía dos prioridades de política exterior: la adhesión a las comunidades europeas y los temas de seguridad (es decir, la renovación del tratado con los Estados Unidos y la cuestión de su ingreso en la OTAN) ${ }^{11}$.

Felipe González ha dicho que los años ochenta fueron la década de la "galopada europea", que comenzó con la ampliación a Grecia en 1980-1981 y prosiguió luego con la formación del eje Bonn-París-Madrid a partir de 1984, con la entrada de España y Portugal en la CEE y la firma del Acta Única de 1986; se sentaban así las premisas fundamentales de la reunificación alemana de 1990 y del Consejo Europeo de Maastricht de 1991². En su esfuerzo de síntesis, esta reconstrucción parece relegar a un segundo plano otros aspectos importantes de la política internacional de aquel periodo: la fase del "europesimismo" de 1980-1984, determinada en buena medida por la así llamada "doctrina europea" de Margaret Thatcher; o -por el contrarioel papel importante que asumió Italia en el proceso de adhesión española a la CEE ${ }^{13}$; o bien, el carácter de los años ochenta como los años de la "segunda guerra fría" entre Oriente y Occidente; o los debates de política exterior de Madrid acerca de si inclinarse más hacia Europa o hacia el área atlántica. El mismo Felipe González no dejó de destacar en diferentes sedes precisamente algunos de estos aspectos, que resultan decisivos para comprender las políticas de los Gobiernos de Madrid en aquellos años.

En este contexto, es necesario recordar también que el 5 de junio de 1980, el presidente francés Giscard d'Estaing había efectuado declaraciones que constituían de hecho casi un veto a nuevas ampliaciones de la CEE. Asimismo, entre 1980 y 1983, Madrid fue la sede de la tercera parte de la Conferencia sobre la Seguridad y la Cooperación en Europa (CSCE), al tiempo que en el plano internacional se hacían evidentes las señales de reanudación del enfrentamiento

9 Marquina Barrio, A. (1986). España en la política de seguridad occidental 1939-1986. Madrid: Ediciones Ejército.

10 Caracciolo, A., \& Bustelo García del Real, C. (1988). Democrazia e sviluppo nella Spagna postfranchista. I problemi della transizione. Milano: FrancoAngeli (pp. 101-148).

11 Mesa, R. (1988). Democracia y política exterior en España. Madrid: EUDEMA; Mugnaini, M. (2002). Le Spagne degli italiani. Milano: Giuffrè (pp. 97-121); Villar, F. (2016). La Transición exterior de España. Del aislamiento a la influencia (1976-1996). Madrid: Marcial Pons (pp. 35-79); Lemus López, E., Pereira Castañares, J. C. (2017). Transición y política exterior (1975-1986). En J. C. Pereira (Coord.), La política exterior de España. De 1800 hasta hoy. 2. ${ }^{\text {a }}$ edición (pp. 659-685). Barcelona: Ariel.

12 González, F., y Cebrián, J. L. (2002). El futuro no es lo que era. Madrid: Punto de lectura (pp. 160-161).

13 Varsori, A. (2010). La Cenerentola d'Europa? L'Italia e l'integrazione europea dal 1947 a oggi. Soveria Mannelli: Rubbettino (pp. 345-352).

\section{Felipe González ha dicho que los años ochenta fueron la década de la "galopada europea", que comenzó con la ampliación a Grecia en 1980- 1981}


bipolar que caracterizó la "segunda guerra fría”. Por lo tanto, la seguridad fue convirtiéndose en un tema cada vez más central de la agenda diplomática de Madrid.

Para España, el año 1981 había sido también un año de dificultades internas: las dimisiones del Gobierno de Adolfo Suárez (19 de enero) y el mandato otorgado por el rey a Leopoldo Calvo Sotelo (12 de febrero), la crisis política del partido de Gobierno (UDC), el intento de golpe de Estado por parte del coronel Tejero (23 de febrero), el terrorismo en el País Vasco, la apertura de nuevos contenciosos entre el Estado y la Iglesia. A todo esto se sumaba el impasse en el terreno diplomático, tanto en la adhesión a las comunidades europeas (mientras que Grecia ya había entrado en enero de 1981) como en los temas relacionados con la seguridad y en especial por el retraso en la renovación del tratado bilateral con los Estados Unidos.

Ante esta situación, el Gobierno de Calvo Sotelo decidió acelerar el proceso de integración de España en la Alianza Atlántica, interpretado también como una carta que jugar para favorecer la entrada de Madrid en la Europa comunitaria. Después de obtener los permisos parlamentarios en 1981, el 29 de mayo de 1982, el ministro de Asuntos Exteriores Pérez-Llorca firmó el instrumento de adhesión de España a la OTAN, con efecto a partir del 30 de mayo. Con España, era la tercera vez que la OTAN ampliaba sus miembros, después de 1952 en que incorporó a Grecia y Turquía, y de 1955 cuando abrió las puertas a la Alemania occidental; la ampliación de 1982 a España fue la última durante la guerra fría ${ }^{14}$.

El año 1982 fue crucial, no solo por la posición internacional de España en el contexto de la "segunda guerra fría”, sino también en el frente interno, donde a las crecientes manifestaciones de la oposición se sumaban las contradicciones internas del partido del Gobierno y el nacimiento de un nuevo partido, el CDS (Centro Democrático Social) liderado por Suárez. En este encendido clima político, a finales de agosto se produjo la disolución de las Cortes y se convocó a elecciones anticipadas para el mes de octubre, en las que se afirmó netamente el PSOE de Felipe González.

En este periodo, la Europa de los Diez veía a España de manera ambivalente: por un lado, no concedía nada en el plano de las tratativas para la adhesión al proceso de integración; por el otro, observaba con preocupado interés el proceso político interno de la España posfranquista para facilitar su consolidación democrática, un proceso que en Europa se veía en términos paralelos a lo que estaba sucediendo en el país vecino, Portugal. Una prueba tangible de esta participación política de los europeos en los asuntos ibéricos eran las nuevas relaciones que se iban instaurando entre los partidos españoles y los principales partidos políticos de Europa (en particular, los partidos representados en el Parlamento europeo elegido por primera vez directamente en 1979). También contribuyó a reforzar la participación de la opinión pública europea en la consolidación democrática de España el movimiento de solidaridad con las nuevas instituciones ibéricas (incluido el rey Juan Carlos) ante el peligro de un retroceso evidenciado por el fallido golpe de 23-F de 1981. En el plano mediático, además, jugó un papel también el resonante éxito de imagen obtenido por España con la organización de la Copa del Mundo de Fútbol de 1982.

Pero hubo otros elementos que contribuyeron a desbloquear las negociaciones para la adhesión española a la CEE. Un papel especial jugó el apoyo del Gobierno de Madrid a la ins-

\section{El Gobierno de Calvo Sotelo decidió acelerar el proceso de integración de España en la Alianza Atlántica, para favorecer la entrada de Madrid en la Europa comunitaria}


talación de los euromísiles norteamericanos en territorio alemán en respuesta a los SS20 soviéticos y la solidaridad surgida en torno a estos temas entre el gobierno de Felipe González y el gobierno alemán de Helmut Kohl, que permitiría vencer las resistencias que aún oponía la Francia de François Mitterrand.

La reactivación del proyecto europeo en los años ochenta aparece como ineludible en el plano histórico: lo destacaron muchos protagonistas de la época subrayando los elementos de novedad y de éxito desde el punto de vista de la política comunitaria, tras las grandes dificultades que atravesó la CEE entre 1973 y 1984, sobre todo, por los efectos de la crisis energética por un lado y de la política de freno adoptada por Gran Bretaña por otro. Dentro de este marco, la adhesión simultánea de Lisboa y Madrid, firmada el 12 de junio de 1985 (vigente desde el 1. ${ }^{\circ}$ de enero de 1986), desempeñó un papel decisivo y constituyó el antecedente inmediato del Acta Única de 1986 (vigente desde el 1. ${ }^{\circ}$ de julio de 1987) que era, a su vez, la primera reforma de los tratados de Roma de 1957 y un verdadero punto de inflexión en la Cooperación Política Europea (CPE). La entrada simultánea de los dos países ibéricos en la CEE fue, por lo tanto, no solo un logro importante para Madrid y Lisboa, sino también un punto de inflexión para toda la política europea.

La puesta en marcha de la Europa de los Doce tenía un significado especial también en la óptica nacional de la política exterior de España. El ingreso de pleno derecho del país ibérico en las comunidades europeas representaba la conclusión de un proceso diplomático largo y tortuoso que había atravesado varias fases, entrelazándose a partir de un determinado momento con la transición democrática de las instituciones nacionales y con la renovación de la política exterior de España. Además, el itinerario de la adhesión española a la Europa comunitaria se entrecruzó continuamente, desde el comienzo hasta la conclusión, con la evolución de la posición del otro país ibérico, Portugal. Todo esto destaca, por un lado, los estrechos lazos entre los diferentes planos de la política europea y, por otro, el significado multifacético, visto desde la perspectiva histórica, de la ruta de adhesión de España a la $\mathrm{CEE}^{15}$.

Este resultado, ansiado por los españoles y conquistado después de una labor diplomática compleja, coincidió con un momento de intensas discusiones sobre el rumbo de la política exterior de España. Fue también el momento de una reorganización del Gobierno ante la dimisión del ministro de Asuntos Exteriores Morán (5 de julio de 1985), reemplazado por Francisco Fernández Ordóñez. Estaba madurando la realización del referéndum sobre la permenencia en la Alianza Atlántica, que fue centro del debate político español en los meses sucesivos. El referéndum español de 12 de marzo de 1986 sobre la permanencia en la OTAN (de cuya estructura militar integrada, España quedaría fuera por el momento ${ }^{16}$ ) se cruzó con los temas europeístas y se convirtió en una especie de referéndum sobre la política exterior del Gobierno. Indudablemente, la entrada de España en la CEE favoreció el exito del sí en el referéndum sobre la permanencia en la OTAN. El resultado favorable no solo despejaba un aspecto crucial para la posición de España en el sistema internacional, sino que anticipaba el resultado de las

15 Bassols, R. (1995). España en Europa. Historia de la adhesión a la CE 1957-85, cit.; Moreno Juste, A. (2000). Las relaciones España/Europa en el siglo XX: notas para una interpretación, cit.; MacLellan, J. C. (2000). Spain and the Process of European Integration, 1957-85, cit.; Mugnaini, M. (2007). Il percorso di adesione della Spagna alla CEE: politica, diplomazia, protagonisti. En G. Angelini, \& M. Tesoro (Eds.), De Amicitia. Scritti dedicati ad Arturo Colombo (pp. 848-860). Milano: FrancoAngeli; Núñez Peñas, V. (2013). Tres escenarios para una negociación: La adhesión de España a la CEE en tiempos de Transición, Reforma y Guerra Fría. Madrid: CEHRI, n. 9.

16 Posición similar a la de Francia en el momento.

La puesta en marcha de la Europa de los Doce tenía un significado especial también en la óptica nacional de la política exterior de España 
inminentes elecciones legislativas (22 de junio de 1986) en las que el PSOE vio ratificada su mayoría absoluta de diputados.

Sucesivamente, los españoles demostraron una notable capacidad de inserción en el mecanismo comunitario y de valorización del rol del país en la construcción europea, así como visión de futuro y determinación al saber captar las ocasiones que ofrecía la CEE (y luego la UE en la posguerra fría), tanto para modernizar y dinamizar la sociedad española como para permitir que el país adquiriera un perfil más alto en el ámbito de la política internacional. Desde el punto de vista de Madrid, el año 1986 cerraba por fin el periodo de transición también en política exterior, al tiempo que, desde el punto de vista de Bruselas, la península ibérica se incorporaba a la construcción de la Europa comunitaria; en el mismo año 1986 los países de la CEE firmaron el Acto Único (17 y 28 de febrero). La convergencia de estas dos perspectivas se advirtió el 1. e de enero de 1989 con el comienzo del primer semestre de presidencia española de la CEE.

Los años ochenta fueron, pues, los de la europeización de la política exterior española y de la incorporación simultánea de los intereses nacionales españoles al contexto de la política europea $^{17}$. Podemos decir que España supo jugar bien la carta europea y, al mismo tiempo, buscó presentarse como una buena carta para Europa en varias oportunidades, en especial como puente en las relaciones con América Latina y entre los protagonistas de las iniciativas con respecto al Mediterráneo (y en particular con el Magreb). Mientras tanto, en los aspectos de política interna, la transición española se erigía como modelo en Europa y fuera de Europa, hasta el punto de ser considerada entre las más logradas de la third wave of democratization reconocida por Samuel Huntington y Juan Linz ${ }^{18}$. En cuanto a la circulación de modelos y de ideas, la España de aquella época estaba de moda, no solo por las dinámicas de modernización económica y política, sino también gracias a fenómenos tales como: la abundante producción literaria, la vivacidad de las iniciativas culturales, la nueva cinematografía, la movida madrileña.

En particular, los años 1989-1992, correspondientes a la transición desde el sistema internacional bipolar a la posguerra fría, representaron una "edad de oro" para la imagen internacional de España, y al mismo tiempo para la percepción optimista de Europa. Como consecuencia de este trayecto y como espejos de la nueva España, debemos recordar los éxitos de imagen de los eventos que fueron organizados en 1992, el llamado Year of Spain ${ }^{19}$ : las XXV Olimpiadas de Barcelona, la Exposición Universal de Sevilla, las celebraciones del Quinto Centenario del Descubrimiento de América y Madrid Capital Europea de la Cultura.

\section{La edad posbipolar: de los años noventa a los retos del nuevo milenio}

Mientras tanto, el cambio geopolítico de los años 1989-1991, con el colapso del bloque soviético y el fin del sistema internacional bipolar, había creado nuevas oportunidades y lanzado nuevos retos a la Europa comunitaria; la primera respuesta fue el tratado de Maastricht (1992) y luego la creación de la Unión Europea. Estas novedades estaban marcando la vida

17 Villar, F. (2016). La Transición exterior de España. Del aislamiento a la influencia (1976-1996), cit., (pp. 81-194).

18 Huntington, S. P. (1991). The Third Wave. Democratization in the late Twentieth Century. Norman-London: University of Oklahoma; Linz, J., \& Stepan, A. (2000). Transizione e consolidamento democratico. Bologna: il Mulino.

19 Closa, C., \& Heywood, P. M. (2004). Spain and the European Union. Basingstoke and New York: Palgrave Macmillan (p. 241).
Los españoles demostraron una notable capacidad de inserción en el mecanismo, así como visión de futuro $y$ determinación al saber captar las ocasiones que ofrecía la CEE 
política de los europeos, con dos tendencias de fondo que caracterizarían los años noventa: por un lado, un viento de optimismo europeo, simbolizado por la conversión de la CEE en UE y la reactivación del proceso de integración europea; por otro, el comienzo de las que más tarde fueron llamadas "nuevas guerras" de la posguerra fría, cuyas manifestaciones más evidentes fueron la primera guerra del Golfo (1990-1991) y las guerras civiles de la ex Yugoslavia (1991-1999). En ambos casos, España buscó una presencia activa, reafirmando el carácter occidental de su propia política exterior.

El Gobierno de Madrid, que en los años ochenta se había puesto al "abrigo" del eje franco-alemán y había ingresado tanto en el club atlántico como en el club europeo, después de la caída del muro de Berlín sostuvo de inmediato la aceleración de la reunificación alemana (1990) respaldada firmemente por el canciller Kohl, incluso ante las incertidumbres de Londres y París. Durante los años que Charles Powell define como the golden years of spanish membership ${ }^{20}$, España buscaba incorporarse desempeñando un papel activo en los diferentes planes de integración europea con la consigna más Europa. Por ejemplo, desarrollando una intensa colaboración con el presidente de la comisión Jacques Delors (1985-1994); entrando a formar parte de la Unión de la Europa Occidental, primero como observador (1988) y luego como miembro de pleno derecho (1990); sosteniendo el mecanismo de la Unión Económica y Monetaria (UEM); participando activamente en las negociaciones de Maastricht (1991-1992); y buscando transformar el format europeo del big four (Alemania, Francia, Gran Bretaña, Italia) en uno más amplio, un big five con la participacion de España. También en lo relacionado con la seguridad europea, España optó por asumir una posición activa, participando en iniciativas como Eurocorps, Eurofor, Euromarfor.

De forma paralela, en 1991 el Gobierno de Madrid decidió participar en la misión internacional de la primera guerra del Golfo, y en el mismo año se eligió la capital española como sede de la Conferencia de paz para Oriente Medio. Por su parte, en 1992 el Consejo Europeo de Lisboa había identificado el Mediterráneo como una de las cuatro zonas de interés de la Política Exterior y de Seguridad Común (PESC) de la naciente UE (las otras tres eran la Europa del Este, Rusia y los Balcanes).

Mientras tanto, la política española sufrió el embate de los problemas económicos del periodo 1992-1994 y la pérdida de la mayoría absoluta del PSOE en las elecciones de 1993: el Gobierno de Madrid pudo sostenerse gracias al apoyo del partido catalán de Jordi Pujol. Esto no impidió que la política exterior continuara siendo activa, característica que mantuvo también durante el periodo en que al frente del Ministerio de Asuntos Exteriores estuvo Javier Solana (de junio de 1992 a diciembre de 1995), que se tradujo en nuevas iniciativas diplomáticas, en particular en las Cumbres Iberoamericanas (la primera tuvo lugar en México en 1991 y la segunda en Madrid en 1992), y en el lanzamiento del proyecto Euro-Med con la conferencia intergubernamental de Barcelona de noviembre de 1995 (durante el semestre de presidencia española de la UE). Dos líneas principales, hacia América Latina y hacia el mundo árabe y la región mediterránea, componentes tradicionales de la política exterior de Madrid que ahora España trataba de incorporar de manera estable en la acción externa de la $U E^{21}$.

20 Powell, C. (2001). Fifteen years on: Spanish membership in the European Union revisited. Center for European Studies at Harvard, Working Paper No. 89, 1-18. Recuperado de: https://ces.fas.harvard.edu/publications/000349fifteen-years-on-spanish-membership-in-the-european-union-revisited

21 Mientras tanto, desde el 1. ${ }^{\circ}$ de enero de 1995, Austria, Suecia y Finlandia también eran miembros de la UE. 
En particular, la Asociación Euro-Mediterránea, también conocida como Barcelona Process o Euro-Mediterranean Partnership, ideada cuando se vislumbraba una posible solución del conflicto palestino-israelí (gracias a los acuerdos de Oslo) y parecía predominar una visión positiva de las relaciones entre la ribera norte y la ribera sur del Mediterráneo. Eran muy sensibles a la nueva posición del área mediterránea en la posguerra fría los países del sur de Europa (España e Italia, pero también Portugal y Grecia) que, de representar la "periferia" de la CEE y el "brazo" de la OTAN en el Mediterráneo, podían llegar a encontrarse en el centro de la gran Europa ampliada. Tras la conclusión de la guerra fría, en especial Francia, Italia y España aspiraban a convertirse en el eslabón de enlace entre la Europa comunitaria y el resto del Mediterráneo. Después de las primeras tentativas del proyecto de diálogo 5+5 para el Mediterráneo occidental, la convergencia de posiciones entre España, Italia y Francia desembocó en la creación del proyecto Euro-Mediterráneo, tema que merecería un tratamiento más profundo porque no se refiere solo a España, obviamente, sino que tuvo repercusiones en las élites políticas y en la opinión pública europea, y va mucho más allá del año $1995^{22}$.

Mas en general, los años noventa fueron un periodo de grandes innovaciones internacionales, que en la perspectiva de la Europa comunitaria vieron nacer varios proyectos e iniciativas que, a veces, se han resumido en la fórmula "sueño europeo" ${ }^{23}$, y que vieron la convergencia de las características de la new wave of regionalism y el "optimismo de la globalización" de ese periodo ${ }^{24}$. España participó en el "sueño euro-optimista” (en cambio los "euro-pesimistas" enfatizaron las guerras de los Balcanes de los noventa), y al mismo tiempo fue un componente importante en varios proyectos regionalistas o interregionales, a partir de la transformación de la CEE en la UE, hasta las iniciativas europeas hacia el Mediterráneo y América Latina.

Mientras tanto Javier Solana, que desde 1982 había sido ministro de todos los Gobiernos españoles ${ }^{25}$, el 5 de diciembre de 1995 fue designado secretario general de la OTAN (cargo que ocupó hasta octubre de 1999) ${ }^{26}$. Al mismo tiempo, ante el creciente desprestigio de la imagen interna del PSOE como consecuencia de una serie de escándalos, hacia finales de 1995 el partido de Jordi Pujol retiró su apoyo al Gobierno. Se convocaron elecciones anticipadas para el 3 de marzo de 1996, en las que se impuso el Partido Popular de José María Aznar. Esta alternancia política era índice de la madurez de la democracia española, pero también constituía un cambio de personal político que fue percibido claramente en los otros países europeos. En algunos casos, hubo quien quiso interpretar este cambio político, que algunos representantes del PP proponían como la segunda transición española, como el nacimiento de un nuevo punto de referencia política para los partidos europeos de centro-derecha.

22 Gillespie, R. (2000). Spain and the Mediterranean: Developing a European Policy towards the South. Houndmills: Macmillan; Calleya, S. C. (2005). Evaluating Euro-Mediterranean Relations. Abingdon and New York: Routledge; Mugnaini, M. (2009). Fasi e percorsi della politica UE verso l'area mediterranea: dalla Conferenza di Barcellona alla Union pour la Méditerranée. En S. Beretta, \& M. Mugnaini (Eds.), Politica estera dell'Italia e dimensione mediterranea (pp. 183-212). Soveria Mannelli: Rubbettino.

23 Sobre este tema elaborado por Jeremy Rifkin ver más en detalle: Moreno Juste, A. (mayo 2011). El proceso de construcción europea y las relaciones España-Europa. En Circunstancia, a.IX, n. 25. Recuperado de: http://www. ortegaygasset.edu/publicaciones/circunstancia/ano-ix---n--25---mayo-2011/articulos/el-proceso-de-construccioneuropea-y-las-relaciones-espana-europa

24 Farrell, M., Hettne, B., \& Van Langenhove, L. (Eds.) (2005). Global Politics of Regionalism. London - Ann Arbor: Pluto Press; Telò, M. (Ed.). (2007). European Union and New Regionalism. Furnham - Burlington: Ashgate.

25 Solana, J., y Bassets, L. (2010). Reivindicación de la política. Veinte años de relaciones internacionales. Barcelona: Editorial Debate.

26 Durante su mandato hubo la ampliación de la OTAN a Polonia, Hungría y República Checa.
Los años noventa fueron un periodo de grandes innovaciones internacionales, que vieron nacer varios proyectos e iniciativas que, a veces, se han resumido en la fórmula "sueño europeo" 
Durante el primer gobierno de Aznar (1996-2000), que nació con el apoyo del partido de Pujol, el cargo de ministro de Asuntos Exteriores fue ocupado por Abel Matutes, que había sido comisario europeo de 1986 a 1994 y luego jefe de la delegación del PP en el Parlamento europeo. Con el Gobierno Aznar-Matutes, prevaleció la idea de continuidad con respecto a la política exterior española de los últimos años, especialmente en lo que respecta a la política europea. Se hizo hincapié especialmente en lograr la unión monetaria (UEM) y en el rechazo de la idea de un núcleo central de la UE que excluyera a España; al mismo tiempo Aznar en 1998 rechazó las propuestas del Gobierno de Roma para aliviar las restricciones de la UEM, sin embargo, el 1. ${ }^{\circ}$ de enero de 1999 tanto Italia como España se encontraban entre los 11 Estados miembros originales de la zona del euro ${ }^{27}$ (de los entonces 15 miembros de la UE) ${ }^{28}$. Otros aspectos que caracterizaron el Gobierno de Aznar fueron la búsqueda de cooperación contra el terrorismo de ETA, el acercamiento al Gobierno británico de Tony Blair en contraste con la anterior alineación de Madrid con el eje franco-alemán, y la mayor importancia otorgada al rol de la OTAN, incluyendo su estructura militar integrada (en la que España ingresó en 1996-1997)29.

Mientras tanto, desde octubre de 1999, Javier Solana cesó la tarea de secretario general de la OTAN y se convirtió en el alto representante de la Política Exterior y de Seguridad Común (PESC) de la UE en la comisión que presidía Romano Prodi (1999-2004), y al mismo tiempo secretario general de la Unión Europea Occidental (UEO-WEU), dos cargos que Solana ocuparía también en 2004-2009 durante el primer mandato de José Manuel Durão Barroso a la presidencia de la Comisión UE ${ }^{30}$.

En las nuevas elecciones políticas españolas de 12 de marzo de 2000, el PP obtuvo la mayoría absoluta de escaños en el Congreso. El éxito, aunque anunciado, superó todas las previsiones y garantizó mayor libertad de acción al segundo Gobierno de Aznar (2000-2004). El notable éxito electoral del PP en las elecciones políticas de 2000 y el énfasis puesto por el Gobierno de Madrid en la liberalización económica, fueron interpretados como un posible nuevo modelo que era menester imitar en otras realidades europeas, por ejemplo, por el centro-derecha en Italia ${ }^{31}$.

Josep Piqué fue designado ministro de Asuntos Exteriores de España. Para lo que concierne a la política exterior, en Europa el segundo Gobierno Aznar acentuó el acercamiento a la Gran Bretaña de Tony Blair y la distancia del eje franco-alemán. Al mayor énfasis puesto en la dimensión nacional de los intereses correspondió luego una orientación siempre creciente hacia el Atlántico y la alineación con los Estados Unidos de la administración de George W. Bush ${ }^{32}$. Después del ataque terrorista de Nueva York de 11 de setiembre de 2001, España participó en la campaña contra el terrorismo internacional y, después de la aprobación por parte de la ONU en diciembre de 2001, también en la intervención internacional en Afganistán.

27 Los otros eran Alemania, Francia, Bélgica, Holanda, Austria, Finlandia, Irlanda, Luxemburgo, Portugal.

28 No eran parte de eso: Grecia (que entraría en la zona del euro en 2001), Gran Bretaña, Dinamarca, Suecia.

29 En la votación parlamentaria de 1996 también el PSOE votó a favor.

30 Como se sabe, después Barroso se mantuvo como presidente de la Comisión UE también en los años 2009-2014.

31 Incisa di Camerana, L. (2000). Il modello spagnolo. Come don Chisciotte è diventato manager. Firenze: Liberal libri.

32 Muñoz-Alonso, A. (2007). España en primer plano. Ocho años de política exterior (1996-2004). Madrid: gotaagota; Iglesias Cavicchioli, M. (2017). Aznar y los 'neocons'. El impacto del neoconservadurismo en la politica exterior de España. Barcelona: Huygens Editorial.

\section{Aspectos que caracterizaron el Gobiemo de Aznar fueron la búsqueda de cooperación contra el terrorismo de ETA y el acercamiento al Gobiemo británico de Tony Blair}


Al mismo tiempo, el Gobierno de Madrid se mostraba preocupado por las nuevas ampliaciones de la UE previstas hacia los países de la Europa central y oriental, temiendo que España quedara relegada a un papel marginal y que también se viera perjudicado el rol de la Asociación Euro-Mediterránea. Mientras tanto, en julio de 2002, fue nombrada ministra de Asuntos Exteriores Ana de Palacio. Ante esta situación, buscando demostrar a la vez protagonismo internacional y alineación con las posiciones angloamericanas, en 2003 el Gobierno Aznar decidió apoyar la intervención militar en Irak, sin la autorización de la ONU, rompiendo políticamente con la Francia de Jacques Chirac y la Alemania de Gerhard Schröder. Es bien sabido que se trató de un momento de fuertes tensiones dentro de la alianza transatlántica y de ásperas divisiones entre los países europeos, que algunos como Donald Rumsfeld trataron de separar entre "vieja” y "nueva” Europa. La España de Aznar, con otros países, fue uno de los protagonistas de esta fractura europea y transatlántica que no se compondría fácilmente. No entraré en detalles sobre este tema, solo quiero mencionar el importante papel desempeñado por la Cumbre de las Azores (16 de marzo de 2003) entre George W. Bush, Tony Blair, José María Aznar y el primer ministro portugués José Manuel Durão Barroso, que ejercía de anfitrión.

Desde varios puntos de vista, el año 2004 marcó un cambio de rumbo. Adoptando la perspectiva de las relaciones internacionales, después de la crisis transatlántica de 2002-2003 el europeísmo y el atlantismo parecieron volver a converger. De echo el año 2004 estuvo marcado más que nada por la "ampliación del Milenio", que vio la entrada de 10 nuevos países en la UE³ (y previendo para 2007 también la entrada de Rumanía y Bulgaria), y por la contemporánea ampliación en 2004 de la OTAN que acogió 7 nuevos miembros ${ }^{34}$.

En España, 2004 fue un año de elecciones políticas, que se celebrarían el 14 de marzo, pero el 11 de ese mismo mes se produjeron los ataques terroristas de Al Qaeda en Madrid: el atentado terrorista más grave que se había registrado en Europa (191 muertos y alrededor de 2000 heridos). El 11-M fue un echo dramático a nivel nacional, pero al mismo tiempo a nivel europeo y mundial, y produjo una fuerte emoción y participación solidaria por parte de los europeos de todas las orientaciones políticas. El modo en el que el Gobierno manejó políticamente este grave hecho fue uno de los factores que contribuyeron a la derrota electoral del candidato del PP en las elecciones del 14 de marzo. El resultado electoral llevaría a la formación del primer Gobierno de José Luis Rodríguez Zapatero (PSOE), que entre sus prioridades tendría la de reconsiderar algunas decisiones de política exterior de los últimos años para regresar a un europeísmo mas tradicional ${ }^{35}$. El 26 de marzo, la reunión del Consejo europeo en Bruselas expresó la solidaridad más sentida por las víctimas, sus familias y todo el pueblo de España.

En el campo de la política exterior del nuevo Gobierno de Madrid, los cambios más rápidos y más perceptibles fueron tres: el retiro de los militares españoles de Irak; el paralelo incremento de la presencia militar de España en la lucha contra el terrorismo islámico en Afganistán; la vuelta a una mayor comprensión con París y Berlín, que se interpretó como un retorno a la ali-

33 Polonia, Estonia, Letonia, Lituania, República Checa, Eslovaquia, Hungría, Eslovenia, Malta, Chipre.

34 Estonia, Letonia, Lituania, Eslovaquia, Eslovenia, Rumanía, Bulgaria.

35 Woodworth, P. (2004, Summer). Spain Changes Course: Aznar's Legacy, Zapatero's Prospects. World Policy Journal, 21(2), 7-26. Recuperado de: http:/www.jstor.org/stable/40209914; Field, B. N. (Ed.) (2011). Spain's 'Second Transition'?: The Socialist Government of José Luis Rodríguez Zapatero. Abingdon and New York: Routledge.

Desde varios puntos de vista, el año 2004 marcó un cambio de rumbo 
neación con el eje franco-alemán ${ }^{36}$. También esta vez, España se convirtió en un modelo para otros países europeos, en este caso, incluso, jugó un papel la solidaridad de los europeos frente a los ataques terroristas del 11-M en Madrid, que fueron interpretados como el primer ataque grave del terrorismo internacional en Europa y contra Europa. Además del valor simbólico, España fue vista como un modelo también de política exterior, sea por las diferentes gestiones de crisis internacionales como la de Irak y la de Afganistán, sea en relación a la política europea.

\section{Conclusiones}

Tomando en consideración todo el periodo 1982-2004 examinado aquîin ${ }^{37}$, podemos destacar varios elementos: algunos positivos, otros negativos, otros que presentan aspectos ambivalentes.

Entre los elementos positivos debe ser subrayado el éxito tanto de la europeización de la política exterior española como del crecimiento del papel internacional de España, con efectos positivos también para la economía y la imagen internacional del país. Un crecimiento del papel de España que probablemente pueda sintetizarse a través de la trayectoria política de un protagonista como Javier Solana que, a pesar de los cambios gubernamentales que ocurrieron en Madrid entre 1982 y 2004, ha sido percibido, a nivel europeo e internacional, como un factor de continuidad en estos años, tanto en las relaciones entre España y Europa, como para el rol de Madrid en la política euroatlántica. Entre los aspectos negativos cabe destacar que el proyecto europeo más ambicioso que tuvo a España como impulsora -junto con otros países de Europa mediterránea como Italia-, la Asociación Euro-Mediterránea de 1995, ingresó en una fase de crisis progresiva (y además el proyecto francés de Union pour la Méditerranée que vino después, realmente no ha despegado $)^{38}$ a partir de las nuevas dinámicas internacionales de 2001-2003 (en particular debido al "arco de crisis" en el Greater Middle East). Entre los elementos ambivalentes se pueden notar los mismos procesos de transformación y ampliación de la CEE-UE, que para España habían jugado un papel estratégico en los años ochenta y hasta mediados de los noventa, sin embargo, los cambios del nuevo milenio (con la ampliación de la UE hacia el este y el lanzamiento de la European Neighbourhood Policy) eran signos de un cambio de prioridades en las políticas de Bruselas que iban a reducir el papel de los países euromediterráneos (y entre ellos España) en la política europea, sacando a la luz el llamado "síndrome periférico"

36 García Cantalapiedra, D. (2014). Spanish foreign policy, the United States and the soft bandwagoning. En D. García Cantalapiedra, \& R. Pacheco Pardo (Eds.), Contemporary Spanish Foreign Policy, cit., (p. 96).

37 Moreno Juste, A. (2000). Las relaciones España/Europa en el siglo XX: notas para una interpretación, cit.; Powell, C. (2001). Fifteen years on: Spanish membership in the European Union revisited, cit.; Royo, S., \& Manuel, P. C. (2003). Spain and Portugal in the European Union. The First Fifteen Years. London and Portland: Frank Cass; Closa, C., \& Heywood, P. M. (2004). Spain and the European Union, cit.; Real Instituto Elcano, European Parliament (2006). 20 Years of Spain in the European Union (1986-2006). Madrid: Real Instituto Elcano; Morata, F., y Mateo, G. (Eds.) (2007). España en Europa - Europa en España (1986-2006). Barcelona: CIDOB; García Lupato, F. (2009). Relevancia y usos de Europa. Efectos directos e indirectos de la Unión Europea sobre la competición política en Italia y España. Barcelona: CIDOB; Moreno Juste, A. (Mayo 2011). El proceso de construcción europea y las relaciones España-Europa, cit.; García Delgado, J. L., Fusi, J. P., y Sánchez Ron, J. M. (2013). España y Europa. Volumen 11 Historia de España - Josep Fontana y Ramón Villares - Directores. Barcelona: Crítica - Marcial Pons (pp. 131-171).

38 Istituto Affari Internazionali, Istituto per gli Studi di Politica Internazionale (2000-2009). L'Italia e la politica internazionale (10 vol. ). Bologna: il Mulino; Gualtieri, R., \& Rhi-Sausi, J. L. (Eds.) (2010). Hub globale, trincea o pantano? Il futuro del Mediterraneo e il ruolo dell'Europa. Rapporto 2010 sull'integrazione europea. Bologna: il Mulino.

\section{Las relaciones} entre España y Europa no tienen lugar en un continente cerrado, sino en un sistema internacional en constante trasformación y que además afecta las relaciones y las percepciones mutuas 
de Madrid frente a la ampliación hacia el este ${ }^{39}$. Otro aspecto ambivalente puede considerarse la compleja interrelación entre la CEE-UE y la dimensión transatlántica de la política europea, y esto (también para España) no solo en el periodo de la guerra fría sino también después, y en particular en los años 2001-2004ㄴ.

En conclusión, en los años ochenta España quería Europa, y tanto la OTAN como las comunidades europeas querían España; en los años noventa, la primera fase de la posguerra fría, España se había convertido en uno de los protagonistas de la política europea y un pilar del "sueño europeo", que vio la convergencia de la new wave of regionalism y el "optimismo de la globalización” de ese periodo; en el nuevo milenio Europa ha fluctuado entre grandes ambiciones y fases de crisis, y España ha compartido estas tendencias. En una perspectiva más amplia, estas consideraciones también pueden servir para recordar que las relaciones entre España y Europa no tienen lugar en un continente cerrado, sino en un sistema internacional en constante trasformación y que además afecta las relaciones y las percepciones mutuas.

\section{Referencias}

Adagio, C., \& Botti, A. (2006). Storia della Spagna democratica. Da Franco a Zapatero. Milano: Bruno Mondadori.

Bariè, O. (2013). Dalla guerra fredda alla grande crisi. Il nuovo mondo delle relazioni internazionali. Bologna: il Mulino.

Bassols, R. (1995). España en Europa. Historia de la adhesión a la CE 1957-85. Madrid: Política Exterior.

Beneyto, J. M., y Pereira, J. C. (Dirs.) (2015). Historia de la política exterior española en los siglos XX y XXI. Volumen I y II. Madrid: CEU Ediciones.

Best, A., Hanhimäki, J. M., Maiolo, J. A., \& Schulze, K. E. (2008). International History of the Twentieth Century and Beyond. $2^{\text {th }}$ ed. London - New York: Routledge. DOI: https://doi. org/10.4324/9780203889862

Bosco, A. (2005). Da Franco a Zapatero. La Spagna dalla periferia al cuore dell'Europa. Bologna: il Mulino.

Calleya, S. C. (2005). Evaluating Euro-Mediterranean Relations. Abingdon and New York: Routledge.

Caracciolo, A., \& Bustelo García del Real, C. (1988). Democrazia e sviluppo nella Spagna postfranchista. I problemi della transizione. Milano: FrancoAngeli.

Closa, C., \& Heywood, P. M. (2004). Spain and the European Union. Basingstoke and New York: Palgrave Macmillan. DOI: https://doi.org/10.1007/978-1-137-05894-2

\footnotetext{
39 Sorroza Blanco, A. (2014). Spain and the European Union. En D. García Cantalapiedra, \& R. Pacheco Pardo (Eds.), Contemporary Spanish Foreign Policy, cit., p. 79.

40 Vacca, G. (Ed.) (2004). Il dilemma euroatlantico. Rapporto 2004 della Fondazione Istituto Gramsci sull'integrazione europea. Bari: Dedalo; Pero, M. del, \& Romero, F. (Eds.) (2007). Le crisi transatlantiche. Continuità e trasformazioni. Roma: Edizioni di Storia e Letteratura; Mammarella, G. (2016). Europa e Stati Uniti dopo la guerra fredda. Bologna: il Mulino.
} 
Farrell, M., Hettne, B., \& Van Langenhove, L. (Eds.) (2005). Global Politics of Regionalism. London - Ann Arbor: Pluto Press.

Field, B. N. (Ed.) (2011). Spain's 'Second Transition'?: The Socialist Government of José Luis Rodríguez Zapatero. Abingdon and New York: Routledge.

García Cantalapiedra, D., \& Pacheco Pardo, R. (Eds.) (2014). Contemporary Spanish Foreign Policy. London and New York: Routledge. DOI: https://doi.org/10.4324/9781315756790

García Delgado, J. L., Fusi, J. P., y Sánchez Ron, J. M. (2013). España y Europa. En Josep Fontana y Ramón Villares (Eds. serie), Historia de España: Vol. 11. Barcelona: CríticaMarcial Pons.

García Lupato, F. (2009). Relevancia y usos de Europa. Efectos directos e indirectos de la Unión Europea sobre la competición política en Italia y España. Barcelona: CIDOB.

Gillespie, R. (septiembre 1997). Northern European Perceptions of the Barcelona Process. Revista CIDOB d'Afers Internacionals, 37, 65-75. Recuperado de http://www.jstor.org/ stable/40585645

Gillespie, R. (2000). Spain and the Mediterranean: Developing a European Policy towards the South. Houndmills: Macmillan. DOI: https://doi.org/10.1057/9780230595675

González, F., y Cebrián, J. L. (2002). El futuro no es lo que era. Madrid: Punto de lectura.

Gualtieri, R., \& Rhi-Sausi, J. L. (Eds.) (2010). Hub globale, trincea o pantano? Il futuro del Mediterraneo e il ruolo dell'Europa. Rapporto 2010 sull'integrazione europea. Bologna: il Mulino.

Huguet, M. (2015). Imágenes y percepciones de España. En J. M. Beneyto, y J. C. Pereira (Dirs.), Historia de la política exterior española en los siglos XX y XXI (pp. 531-557). Volumen I. Madrid: CEU Ediciones.

Huntington, S. P. (1991). The Third Wave. Democratization in the late Twentieth Century. Norman-London: University of Oklahoma.

Incisa di Camerana, L. (2000). Il modello spagnolo. Come don Chisciotte è diventato manager. Firenze: Liberal libri.

Istituto Affari Internazionali, Istituto per gli Studi di Politica Internazionale (2000-2009). L'Italia e la politica internazionale (10 vols). Bologna: il Mulino

Jones, A. (Jun. 2006). Narrative-Based Production of State Spaces for International Region Building: Europeanization and the Mediterranean. Annals of the Association of American Geographers, 96(2), 415-431. Recuperado de: http://www.jstor.org/stable/3694054

Kausch, K., \&Y oungs, R. (Sep. 2009). The End of the 'Euro-Mediterranean Vision'. International Affairs (Royal Institute of International Affairs 1944-), 85(59), 963-975. Recuperado de http://www.jstor.org/stable/40388918

Iglesias Cavicchioli, M. (2017). Aznar y los 'neocons'. El impacto del neoconservadurismo en la política exterior de España. Barcelona: Huygens Editorial.

Leffler, M. P., \& Westad, O. A. (Eds.) (2010). The Cambridge History of the Cold War. (3 vols.). Cambridge: Cambridge University Press. DOI: https://doi.org/10.1017/ CHOL9780521837194 
Linz, J., \& Stepan, A. (2000). Transizione e consolidamento democratico. Bologna: il Mulino

Lundestad, G. (Ed.) (2013). International Relations Since the End of the Cold War. Oxford: Oxford University Press.

MacLellan, J. C. (2000). Spain and the Process of European Integration, 1957-85. New York: Palgrave. DOI: https://doi.org/10.1057/9781403932679

Marquina Barrio, A. (1986). España en la política de seguridad occidental 1939-1986. Madrid: Ediciones Ejército.

Mammarella, G. (2016). Europa e Stati Uniti dopo la guerra fredda. Bologna: il Mulino.

Mesa, R. (1988). Democracia y politica exterior en España. Madrid: EUDEMA.

Morata, F. y Mateo, G. (eds.) (2007). España en Europa - Europa en España (1986-2006). Barcelona: CIDOB.

Morelli, U. (2011). Storia dell'integrazione europea. Milano: Guerini.

Moreno Juste, A. (2000). Las relaciones España/Europa en el siglo XX: notas para una interpretación. Cuadernos de Historia Contemporánea (22), 95-133.

Moreno Juste, A. (Mayo 2011). El proceso de construcción europea y las relaciones EspañaEuropa. Circunstancia, (25).

Moreno Juste, A. (2015). España en Europa: sistemas internacionales, política exterior y construcción europea. En J. M. Beneyto, y J. C. Pereira (Dirs.), Historia de la política exterior española en los siglos XX y XXI (pp. 619-651). Volumen I. Madrid: CEU Ediciones.

Moreno Juste, A., \& Núñez Peñas, V. (2017). Historia de la construcción Europea desde 1945. Madrid: Alianza.

Mugnaini, M. (2002). Le Spagne degli italiani. Milano: Giuffrè.

Mugnaini, M. (2007). Il percorso di adesione della Spagna alla CEE: politica, diplomazia, protagonisti. En G. Angelini, \& M. Tesoro (Eds.), De Amicitia. Scritti dedicati ad Arturo Colombo (pp. 848-860). Milano: FrancoAngeli.

Mugnaini, M. (2009). Fasi e percorsi della politica UE verso l'area mediterranea: dalla Conferenza di Barcellona alla Union pour la Méditerranée. En S. Beretta, \& M. Mugnaini (Eds.), Politica estera dell'Italia e dimensione mediterranea (pp. 183-212). Soveria Mannelli: Rubbettino.

Muñoz-Alonso, A. (2007). España en primer plano. Ocho años de política exterior (1996-2004). Madrid: gotaagota.

Núñez Peñas, V. (2013). Tres escenarios para una negociación: La adhesión de España a la CEE en tiempos de Transición, Reforma y Guerra Fría. Madrid: CEHRI, n. 9.

Núñez Seixas, X. M. (Coord.) (2017). España en democracia, 1975-2011. En Josep Fontana y Ramón Villares (Dirs. serie), Historia de España: Vol. 10. Barcelona: Crítica - Marcial Pons.

Pereira Castañares, J. C., \& Moreno Juste, A. (1991). La Spagna franchista di fronte al processo di costruzione europea (1945-1970). En Storia delle relazioni internazionali, a. 7, n. 1, 54-88. 
Pereira, J. C. (Coord.) (2003). Historia de las relaciones internacionales contemporáneas. 2. ${ }^{\text {ed. }}$ Barcelona: Ariel.

Pereira, J. C. (Coord.) (2017). La politica exterior de España. De 1800 hasta hoy. 2. ${ }^{\text {a }}$ ed. Barcelona: Ariel.

Pero, M. del, \& Romero, F. (Eds.) (2007). Le crisi transatlantiche. Continuità e trasformazioni. Roma: Edizioni di Storia e Letteratura.

Powell, C. (2001). España en democracia, 1975-2000. Barcelona: Plaza \& Janes.

Powell, C. (2001). Fifteen years on: Spanish membership in the European Union revisited. Center for European Studies at Harvard, Working Paper No. 89, 1-18. Recuperado de https://ces.fas.harvard.edu/publications/000349-fifteen-years-on-spanish-membershipin-the-european-union-revisited

Prego, V. (2000). Presidentes. Veinticinco años de historia narrada por los cuatro jefes de Gobierno de la democracia. Barcelona: Plaza \& Janes.

Real Instituto Elcano, European Parliament. (2006). 20 Years of Spain in the European Union (1986-2006). Madrid: Real Instituto Elcano.

Royo, S., \& Manuel, P. C. (2003). Spain and Portugal in the European Union. The First Fifteen Years. London and Portland: Frank Cass.

Smith, M. (2000). NATO Enlargement during the Cold War. Basingstoke and New York: Palgrave Macmillan. DOI: https://doi.org/10.1057/9780333985359

Solana, J., y Bassets, L. (2010). Reivindicación de la política. Veinte años de relaciones internacionales. Barcelona: Editorial Debate.

Telò, M. (Ed.) (2007). European Union and New Regionalism. Furnham - Burlington: Ashgate.

Tusell, J. (Coord.) (2003). La transición a la democracia y el reinado de Juan Carlos I. En José María Jover Zamora (Dir.) Historia de España Menéndez Pidal: Tomo XLII. Madrid: Espasa Calpe.

Vacca, G. (Ed.) (2004). Il dilemma euroatlantico. Rapporto 2004 della Fondazione Istituto Gramsci sull'integrazione europea. Bari: Dedalo.

Varsori, A. (2010). La Cenerentola d'Europa? L'Italia e l'integrazione europea dal 1947 a oggi. Soveria Mannelli: Rubbettino.

Villar, F. (2016). La Transición exterior de España. Del aislamiento a la influencia (1976-1996). Madrid: Marcial Pons. DOI: https://doi.org/10.2307/j.ctt20fw785

Woodworth, P. (2004, Summer). Spain Changes Course: Aznar's Legacy, Zapatero's Prospects. World Policy Journal, 21(2), 7-26. Recuperado de http://www.jstor.org/stable/40209914 